\title{
Interview with Omer Biran and Michael Halfmann on "Conversational Agents at SAP"
}

\author{
Jella Pfeiffer
}

Published online: 14 April 2020

(c) Springer Fachmedien Wiesbaden GmbH, ein Teil von Springer Nature 2020

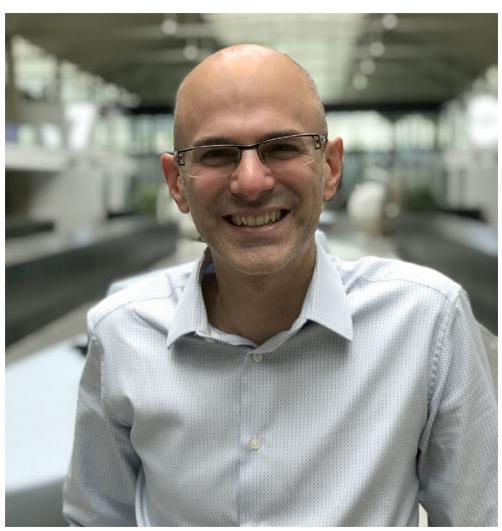

Omer Biran

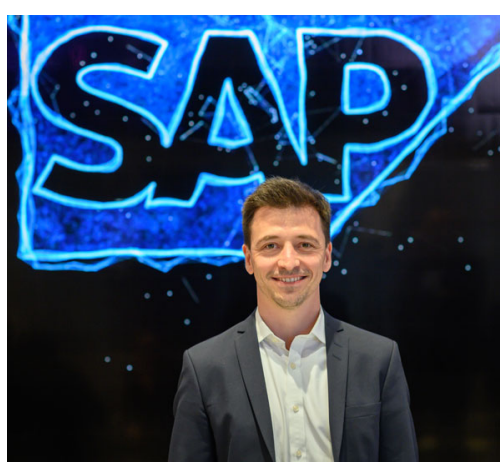

Michael Halfmann

J. Pfeiffer $(\bowtie)$

Chair of Digitalisation, E-Business and Operations Management, Justus Liebig University Giessen, Licher Str. 74, 35394 Giessen, Germany

e-mail: jella.pfeiffer@wirtschaft.uni-giessen.de
Omer Biran is Conversational AI's Managing Director and Michael Halfmann is Head of Intelligent Enterprise CoE (Center of Excellence) at SAP. Omer Biran brings new interfaces to the intelligent enterprise in order to provide intelligent and easy to interact services to employees. Michael Halfmann is responsible for the implementation of conversational agents that support internal SAP processes.

BISE: In recent years, advances in technology have facilitated new forms of intelligent assistance systems. What makes these systems 'intelligent' and how does this create value for SAP?

Omer: We live in a world where users expect interfaces to be smooth, instantaneous and mobile. SAP strives to give customers such an experience. We want to ensure that our customers do not run into low productivity or frustration when using SAP products. One solution to improve the user experience is to simplify the navigation in our rich and complex software and automate repetitive tasks. And to do this, chatbots are an excellent solution.

By augmenting all SAP products with powerful conversational interfaces that understand human language, we are allowing all our customers to become the intelligent enterprise, simplify the life of their employees and customers, and be more efficient every day.

Conversational interfaces are made intelligent by their ability to understand human language. As a manager, if you want to create a new hiring position, you need to go on your HR tool, struggle to find the right page, and then start filling the form. With conversational interfaces, you simply need to say "I want to hire a new personal assistant", and the right page opens up for you with the job title pre-filled!

That's what we are achieving currently at SAP. 
BISE: At SAP you offer intelligent user assistance systems to your employees in form of Conversational Agents (CAs). Which tasks do these agents fulfill? For which kind of services and processes are they particularly helpful?

Michael: We offer conversational agents for various areas and processes, from sales and IT support over assistance with procurement and contracts to daily employee requests such as campus navigation or lunch menu display. Generally, the individual assistants can be classified into two categories:

1. General product bots: These are dynamic bots built by SAP products to improve the user experience of the SAP portfolio. These bots understand all the topics that an employee can mention and can direct them to the right page or execute simple actions on their behalf. These bots are powered by the entire business systems of SAP and can access complex information easily to dispatch it in a simple way. Common examples include "Can you show me my pending approvals", "can I take a day off" or "I want to create a new position for an employee".

2. Simple bots: These are rather static bots that can perform very simple tasks or answer FAQs, such as "what is for lunch today" or "what is the exact address of the SAP offices in Germany?"

Building bots that are increasingly complex requires a powerful technology on language management (understanding human language) and on the dialog management (creating smooth conversation flows). We see ever growing demand for additional bots to be added from all areas. The demand also gets more complex leading to more and more transactional tasks being completed by conversational assistants in the near future.

BISE: What do you hope to gain from the introduction of CAs in general? And what potential do you see in analyzing the usage data in order to improve business processes?

Michael: We hope to gain, or partially already do gain, in multiple aspects. The first aspect is a drastic improvement of the user experience. This means that users are much more efficient when using the interface and have access to information much more easily. They are more at ease with our software.

The second aspect would be productivity. With chatbots automating simple tasks like support, users can refocus their work on higher value tasks.

By analyzing the input from all users with clustering and unsupervised learning techniques, we gain insights on where there is demand for further extension of the conversational capabilities as well as insights on where processes might not be designed in an ideal way. Indirectly, the users basically tell us directly, in natural language, what they need - we only need to get the analysis right and react accordingly.

BISE: When did you launch the first CA in your company and what were your first impressions when launching the CAs? Have they been adopted yet by many employees and who would be the typical user for early adoption?

Michael: Our first internally launched bot was the IT support bot. This is somehow the natural first step, since in IT we have all the technical people who like trying new technologies and bringing them to actual productive use. The bot went live in 2017 almost 3 years ago. Meanwhile the IT support bot is the central entry point to IT support, all requests go to the bot first. The adoption has increased significantly over the years and is meanwhile on a mainstream level. The early adopters are typically technically interested people who are keen on experimenting with new technology.

BISE: Interactions between employees and CAs inherently involve human-computer interaction. Can you tell us more about the process you engage into design this interaction?

Omer: Our extensive experience with customers over the past four years have allowed us to learn what makes a good conversational interface. There are two things we pay extra attention on to create a great experience: "Horizontal coverage" and short exchanges.

"Horizontal coverage" is essential for a good humanmachine interaction. The goal of this methodology is to build a chatbot that understands everything the user is saying and to avoid the infamous "I'm sorry, I did not understand, can you rephrase?". To achieve that, we train SAP chatbots with sentences that we gather from real end users. It encapsulates everything users can possibly say and will allow the chatbot to understand everything. After understanding the input, the chatbot will either execute the user request, or in case of a complex request, the chatbot will navigate the user to the right page in the SAP solution for the user to be able to complete the process. The value is straightforward - the user never gets stuck.

Conversations with machines work their best on small exchanges. When designing bots, we advise teams to honor the "reward principle": after 2-3 replies from the user, the bot has to give the user a reward. A reward can be a first bit of information, navigation or redirection to appropriate content. That way, three sentences in, the user already has gained value from the interaction.

BISE: Are the agents yet capable of fully executing these tasks? To what extent are service employees still involved in answering the requests and fulfilling the tasks? 
Michael: This is highly dependent on the kind of task an agent is supposed to solve. First level support queries can be fully answered by conversational agents. When it gets to more complex requests, typically answered by expert users from second or third level support, we still have human agents to step in. In transactional scenarios this is also a typical pattern. A conversational agent can perform the tasks it was trained/implemented to do and can combine input parameters dynamically. However, once it comes to unforeseen requests the bot will not be able to fully cover the requested execution. With Machine Learning techniques a certain flexibility can be achieved but there are certainly still limits where a human has to step in.

A very powerful scenario which we are currently ramping up, is the combination of Conversational AI with Intelligent Robotic Process Automation. Robotics Process Automation (RPA) is a common technology to automate human interaction with software by scripts which basically steer the software through UI and API interaction. Combined with conversational agents such process automation scripts can be triggered through conversational AI based agents. On one hand this allows for changes in the process flow without having to retrain the conversational agent whereas on the other hand it still hides the process complexity from the end user.

BISE: Where do CAs still struggle and which are the next steps for improvement?

Michael: For our internal setup of multiple conversational agents we have a central entry point through which end users access all agents. The idea is to improve the user experience by selecting the right bot to answer a specific request based on the request. This step however, selecting the right agent to answer a given request, is quite challenging and gets more difficult the more bots we connect to the central multibot manager.

Omer: Our goal is to augment all SAP products with powerful conversational interfaces. To do this, we want to go even further on low code system integration, bot delivery, and development experience.

Integrating your bot in back-end systems is essential to provide an integrated experience. Our mission is to remove the burden of developing complex code interfaces for bot developers, and to offer the capacity to easily manage the connection in our bot building tool.

Bot delivery is key to allow each customer their own experience. While SAP will offer conversational interfaces for all SAP lines of business, we know each customer is different. Therefore, we will allow the customization of all SAP standard bots to our customers. We'll also work on enabling a very convenient setup of multiple chatbots through one unique entry point for users to further simplify the interaction with CAs, directly targeting the challenge Michael just mentioned. This is one of our big topics for 2020 !

Finally, we are always striving to make bot building as simple as possible for bot developers. That goes through a simple UX or integrated development tools like advanced version control, unit testing and insights gathering. We are also going to keep focusing on Bot Generators, tools that generate full-fledged bots from data sources like policy documents, web-services (OData) or databases!

BISE: Which technology do you use for the implementation? Are they text-based or also voice based?

Michael: For our internal setup we are leveraging our own SAP Conversation AI platform with one digital assistant as entry point on the central Fiori Launchpad as well as through a native iOS app.

The bots are defined text-based, but an optional voice recognition capability is connected before the input which then gets translated into text for further processing. Once the natural language understanding module from the Conversational AI platform has identified the user's intent the reply is defined as a combination of text, data retrieved through API calls and/or robotics process automation execution.

Between the end-user's input and the individual bot answering we have a multibot manager which decides on the right bot to answer. The distribution is based on the bots' confidence and further options, like letting the enduser choose the right bot in case the situation is not clear enough.

BISE: Are the CAs linked to and/or complemented with other intelligent user assistance systems?

Michael: Yes, most CAs, except for very simple ones, have some sort of integration with other systems. Most integrate via APIs into the backend business systems for data exchange or to trigger certain actions. Some of our internal bots also integrate into machine learning based systems to base their behavior on certain parameters like the organizational position of the requestor.

Recently SAP has implemented a standard connection between conversational agents built with SAP Conversational AI and its Robotic Process Automation platform. We have started leveraging this new option for internal bots and see great potential in the extension of this possibility to simplify business processes in most areas.

BISE: Do you also offer CAs in your products or plan to do so? Where do you see the largest potential?

Omer: Indeed, we do. The mission of SAP Conversational is to bring conversational to the intelligent enterprise. SAP products are extremely powerful, but in this efficiency, 
there can be a complexity that impacts our end-users. We want to simplify the experience of all SAP users. Conversational interfaces are the best way to combine the powerful software with an efficient and smooth interface.
Therefore, we are currently building powerful CAs for the major SAP products.

BISE: Thank you very much for your time and for this interview. 